\title{
Somatosensory Findings of Pusher Syndrome in Stroke Patients
}

\author{
Jong Hwa Lee, MD, Sang Beom Kim, MD, Kyeong Woo Lee, MD, Ji Yeong Lee, MD \\ Department of Physical Medicine and Rehabilitation, Dong-A University College of Medicine and \\ Regional Cardiocerebrovascular Center, Dong-A University Hospital, Busan, Korea
}

Objective To investigate the somatosensory findings of pusher syndrome in stroke patients.

Methods Twelve pusher patients and twelve non-pusher patients were enrolled in this study. Inclusion criteria were unilateral stroke, sufficient cognitive abilities to understand and follow instructions, and no visual problem. Patients were evaluated for pusher syndrome using a standardized scale for contraversive pushing. Somatosensory finding was assessed by the Cumulative Somatosensory Impairment Index (CSII) and somatosensory evoked potentials (SEPs) at 1 and 14 weeks after the stroke onset. Data of SEPs with median and tibial nerve stimulation were classified into the normal, abnormal, and no response group.

Results In the baseline characteristics (sex, lesion character, and side) of both groups, significant differences were not found. The score of CSII decreased in both groups at 14 weeks $(p<0.05)$, but there were no significant differences in the CSII scores between the two groups at 1 and 14 weeks. There were no significant differences in SEPs between the two groups at 1 and 14 weeks after the stroke onset.

Conclusion It appears that somatosensory input plays a relatively minor role in pusher syndrome. Further study will be required to reveal the mechanism of pusher syndrome.

Keywords Pusher syndrome, Stroke, Somatosensory evoked potentials, Cumulative Somatosensory Impairment Index

Received May 31, 2012; Accepted September 17, 2012

Corresponding author: Ji Yeong Lee

Department of Physical Medicine and Rehabilitation, Dong-A University College of Medicine, 32 Daesingongwon-ro, Seo-gu, Busan 602-714, Korea

Tel: +82-51-240-5690, Fax: +82-51-254-8511, E-mail: easy0318@naver. com

(ㄷ) This is an open-access article distributed under the terms of the Creative Commons Attribution Non-Commercial License (http://creativecommons. org/licenses/by-nc/3.0) which permits unrestricted noncommercial use, distribution, and reproduction in any medium, provided the original work is properly cited.

Copyright $\odot 2013$ by Korean Academy of Rehabilitation Medicine

\section{INTRODUCTION}

Stroke is a critical disease associated with high morbidity and mortality rates, as seen in the Republic of Korea as well as other developed nations [1]. After stroke, patients often encounter disturbances of balance and postural control. They fail to maintain an appropriate posture against gravity, or their gravity line shifts toward the nonparetic side in the standing position as a result of the muscle tone change, hypoesthesia, and perceptual dysfunction caused by hemiplegia [2,3]. In contrast, some patients use the unaffected arm or leg to actively push away from the unparalyzed side and resist any attempts to passively correct their tilted body posture; this 
phenomenon is known as pusher syndrome (PS) [4,5]. PS causes several problems in the performance of independent and functional activities of daily living (ADL) and delays the recovery of motor ability, thereby acting as a barrier to rehabilitation [6].

According to previous studies, the prevalence rate of PS varies widely, and it is a transient phenomenon that recovers within several weeks in the majority of stroke patients $[7,8]$. In many cases, PS is frequently associated with proprioceptive disorder and cognitive dysfunctions, such as hemineglect and aphasia [7,9].

The pusher behavior usually occurs when the patient is in an upright position. It is known to occur because of the misperception of the body orientation in relation to gravity. When seated in a chair with their eyes closed, the patients perceive their body as oriented upright when they are actually tilted by nearly $20^{\circ}$ to the side, and when they open their eyes, their posture returns to normal [6]. Bisdorff et al. [10] showed that humans depended on proprioception to maintain an upright posture, and Karnath [6] noted that the somatic senses were one element of the sensory inputs in the gravity receptor system.

Although PS is occasionally observed in the stroke patients, the research on this syndrome in relation to the balance and postural control disturbances is insufficient. Some previous studies $[6,11]$ have shown that somatosensory loss does not necessarily result in PS, with the results being based on subjective experiments. Therefore, more extensive research is necessary to clarify the vertical perception bias in patients with PS [12]. In this study, we examined the somatic senses by the electrodiagnostic study and somatosensory impaired index in PS patients and tried to establish the relation between the somatosensory impairment and PS.

\section{MATERIALS AND METHODS}

\section{Subjects}

The study prospectively enrolled 131 patients who were admitted with stroke at the author's hospital between May 2010 and August 2011. The patients were diagnosed with stroke on the basis of history, systemic observation, neurological examination, and imaging studies (computed tomography or magnetic resonance imaging). The experimental group included 12 stroke patients with PS (3 with cerebral hemorrhage and 9 with cerebral infarc- tion), selected from 114 patients (42 with cerebral hemorrhage and 72 with cerebral infarction). Patients who presented with a prior history of stroke, did not receive rehabilitation treatment owing to rapid recovery, and did not wish to collaborate were excluded. The patients who had impaired visuospatial function, and had an unstable medical condition were also excluded. The control group comprised 12 non-PS patients ( 4 with cerebral hemorrhage and 8 with cerebral infarction). The mean age of the patients was $65.9 \pm 3.9$ years. There were 13 male and 11 female patients. Of the 24 stroke patients, 15 were right-sided hemiplegia, and 9 were left-sided hemiplegia.

\section{Methods}

We determined whether the subjects developed PS within 1 week after hospitalization. Patients were evaluated for PS using a standardized scale for contraversive pushing. Somatosensory finding was assessed by $\mathrm{Cu}$ mulative Somatosensory Impairment Index (CSII) [13] and somatosensory evoked potentials (SEPs) at 1 and 14 weeks after the stroke onset. Patients with PS were assigned to the experimental group (group I), and those without PS to the control group (group II).

\section{Evaluation of the presence of PS and its severity}

The method proposed by Karnath et al. [14] was used to determine whether the subjects had PS and the severity of the syndrome, if present. PS was diagnosed when the subjects exhibited all following three criteria with a minimum score of 1 for each item: 1 ) the symmetry of spontaneous body posture; 2) the use of nonparetic extremities (leg or arm) to push by abduction and extension thrust; and 3) the resistance to passive correction of tilted posture. The severity of the condition in the PS patients was measured using the Scale for Contraversive Pushing (SCP), standardized by Karnath et al. [14].

\section{Somatosensory evoked potentials}

SEPs is mostly conducted along the posterior funiculi and medial lemnisci. This test was performed at 1 week after the stroke onset. The Viking Select electromyography (EMG) system (Nicolet Biomedical Inc., Lilburn, GA, USA) was used, and the temperature of the testing room was maintained between $22^{\circ} \mathrm{C}$ and $25^{\circ} \mathrm{C}$. With the subject lying on a bed in a relaxed position, the median nerves of both wrists and the tibial nerves of both ankles were 
stimulated, and the recordings were made from the scalp electrodes. The minimum stimulation intensity was determined as the intensity at which the slight movement of the thumbs and great toes were grossly observed. The stimulation frequency was 3 times per second, the results of 100-200 repetitive stimulations were averaged, and the test was performed twice. The stimulation duration time was $0.1 \mathrm{~ms}$. The recording speed was set at $5 \mathrm{~ms} /$ division for the upper extremities and $10 \mathrm{~ms} /$ division for the lower extremities, and the recording sensitivity was set at $5 \mu \mathrm{V} /$ division. For median nerve evaluation, active electrodes were placed at C3' and C4', and the reference electrode was placed at Fz. For tibial nerve assessment, active electrode was placed at Cz', and the reference electrode was placed at Fz. Comparison of evoked potentials on the paretic and nonparetic sides was made, and based on the results, the subjects were divided into the normal group, abnormal group, and no response group. When the amplitude on the paretic side was less than $50 \%$ that of the nonparetic side, or the delayed latency on the paretic side was $10 \%$ more than that of the nonparetic side, patient was assigned to the abnormal group. When no potential was triggered, the patient was assigned to the no response group.

\section{Cumulative Somatosensory Impairment Index}

Participants assumed a supine position with their eyes closed. The CSII for the lower limbs was derived from the clinical tests of pressure and vibration sensitivity, ankle proprioception, and graphesthesia. Pressure sensitivity was tested using 4.31 and 4.56 Semmes-Weinstein monofilaments applied to the skin of the external malleolus. Each filament was applied twice, and the sensitivity for that filament was considered absent if it was not sensed

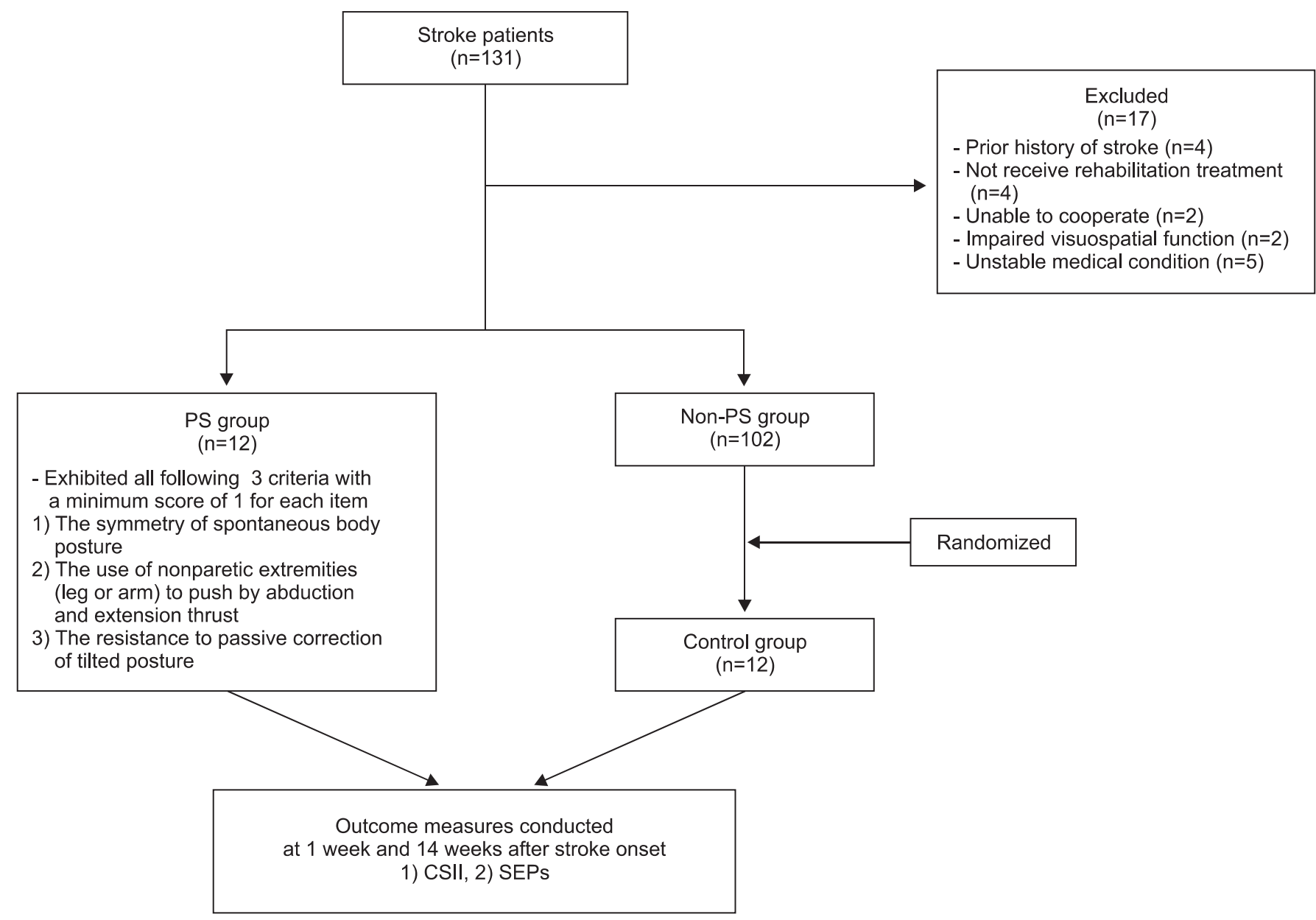

Fig. 1. The enrollment algorithm for the subjects from May 2010 to August 2011 . The 24 patients included in the study were among the 131 stroke patients. PS, pusher syndrome; CSII, Cumulative Somatosensory Impairment Index; SEPs, somatosensory evoked potentials. 
on at least one trial. Pressure sensitivity was recorded as follows: 0 , normal (both filaments sensed); 1 , reduced (only 4.56 filament sensed); and 2, absent (neither filament sensed). Graphesthesia was evaluated by asking participants to recognize 3 simple symbols (line, circle, plus sign) that were drawn on their sole. Graphesthesia was rated as follows: 0 , normal (all 3 correct); 1 , reduced ( 1 error); and 2, absent ( $\geq 2$ errors). For testing proprioception, the examiner positioned the reference ankle randomly at $10^{\circ}$ dorsiflexion and $20^{\circ}$ plantarflexion, from the neutral position. The participant was asked to place the test ankle in the same position. The proprioception was considered absent if in both positions the error of the tested ankle was greater than $5^{\circ}$ of the reference ankle. It was considered reduced if this error occurred only in 1 of the 2 positions. Proprioception was rated as follows: 0 , normal; 1 , reduced; and 2, absent. The vibratory stimulus was created with a $128-\mathrm{Hz}$ diapason on the bony prominence of the first metatarsal bone. The participants were asked to report if they perceived the vibration. If the vibration was perceived, they were asked to indicate when they no longer perceived the vibration. Vibration sensitivity was graded as follows; 0 , normal ( $\geq 10 \mathrm{sec}$ ); 1 , reduced (1-9 sec); and 2, absent. Total CSII scores ranged from 0 to 8 , with 8 being the worst.

\section{Statistics}

The data was statistically analyzed using SPSS ver. 14.0 (SPSS Inc., Chicago, IL, USA). A comparative analysis

Table 1. Basic characteristics between two groups

\begin{tabular}{|c|c|c|c|}
\hline Characteristic & $\begin{array}{c}\text { Group I } \\
(n=12)\end{array}$ & $\begin{array}{c}\text { Group II } \\
(\mathrm{n}=12)\end{array}$ & $\begin{array}{c}\mathbf{p}- \\
\text { value }\end{array}$ \\
\hline Age (yr), mean \pm SD & $65.4 \pm 4.7$ & $66.5 \pm 3.1$ & 0.594 \\
\hline Sex (male/female) & $7 / 5$ & $6 / 6$ & 0.682 \\
\hline Infarction, no. (\%) & $9(75)$ & $8(66.6)$ & 0.653 \\
\hline Affected side (right/left) & $8 / 4$ & $7 / 5$ & 0.673 \\
\hline \multicolumn{4}{|c|}{ Paresis of contralesional side $\mathrm{e}^{\mathrm{a})}$} \\
\hline Arm, median (range) & $1.0(0-3)$ & $1.5(0-4)$ & 0.424 \\
\hline Leg, median (range) & $2.5(0-3)$ & $3.0(1-4)$ & 0.528 \\
\hline Initial K-MBI, mean \pm SD & $31.8 \pm 8.1$ & $34.9 \pm 4.5$ & 0.217 \\
\hline
\end{tabular}

SD, standard deviation; K-MBI, Korean Modified Barthel Index; Group I, patients with pusher syndrome; Group II, patients without pusher syndrome.

${ }^{\text {a) }}$ Paresis of the contralateral arm and leg was scored with the usual clinical ordinal scale, where ' 0 ' stands for no trace of movement and ' 5 ' for normal movement. between the experimental group and control group was conducted using the chi-square test, with items including the gender, lesions, types of stroke, and initial characteristics. An independent t-test was used to identify significant differences between the groups in CSII. A paired t-test was used to compare CSII between 1 and 14 weeks. We used the chi-square test to identify significant differences between the groups in SEPs result. A p-value $<0.05$ was considered statistically significant.

\section{RESULTS}

Participant flow through the trial is described in Fig. 1. In the baseline characteristics (age, sex, lesion, initial strength, and initial Korean modified Barthel index [KMBI]) of both groups, significant differences were not found (Table 1).

\section{SCP score changes in both groups}

Fig. 2 shows the status of pusher symptoms on the three SCP subscales at 1 and 14 weeks. At 1 week after stroke onset, all PS patients showed severe contraversive pushing on each of the SCP subscales. However, they showed reduction in the symptoms and the scores of PS at 14 weeks (Fig. 2A). None of the control group showed fulfilled clinical criterion to be diagnosed as contraversive pushing (Fig. 2B).

\section{Comparison of CSII}

No significant difference was found in the CSII scores between the experimental and control groups at 1 week after the stroke onset (Table 2). Similarly, no significant difference was found in the CSII scores between the two groups at 14 weeks after the stroke onset (Table 2). However, the change of CSII scores between 1 and 14 weeks were significantly different. The CSII score significantly

Table 2. Comparison of CSII between two groups

\begin{tabular}{lccc}
\hline & Group I & Group II & p-value \\
\hline 1 week CSII & $5.7 \pm 1.6$ & $4.6 \pm 1.1$ & 0.215 \\
14 weeks CSII & $2.4 \pm 0.5$ & $1.9 \pm 0.8$ & 0.312 \\
p-value & $0.032^{*}$ & $0.024^{*}$ & \\
\hline
\end{tabular}

Values are presented as mean \pm standard deviation. Group I, patients with pusher syndrome; Group II, patients without pusher syndrome; CSII, Cumulative Somatosensory Impairment Index.

${ }^{*} \mathrm{p}<0.05$. 
(A)

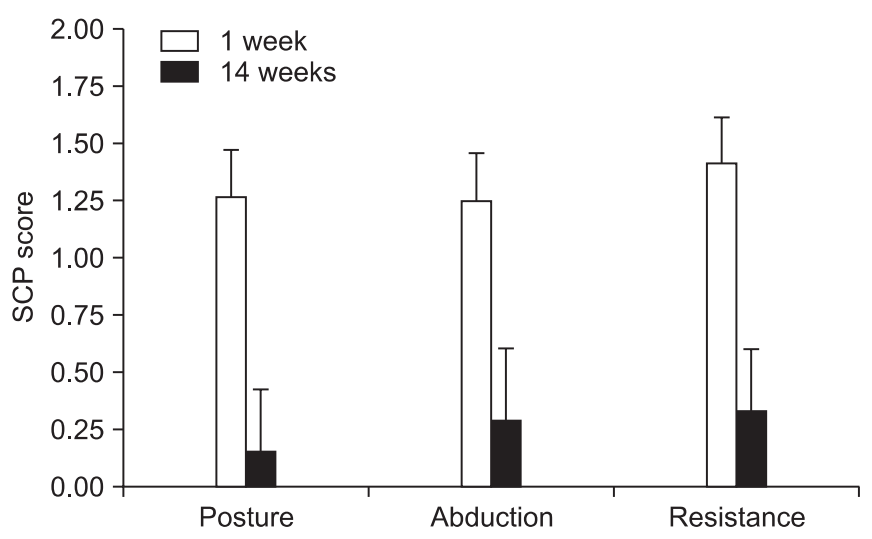

(B)

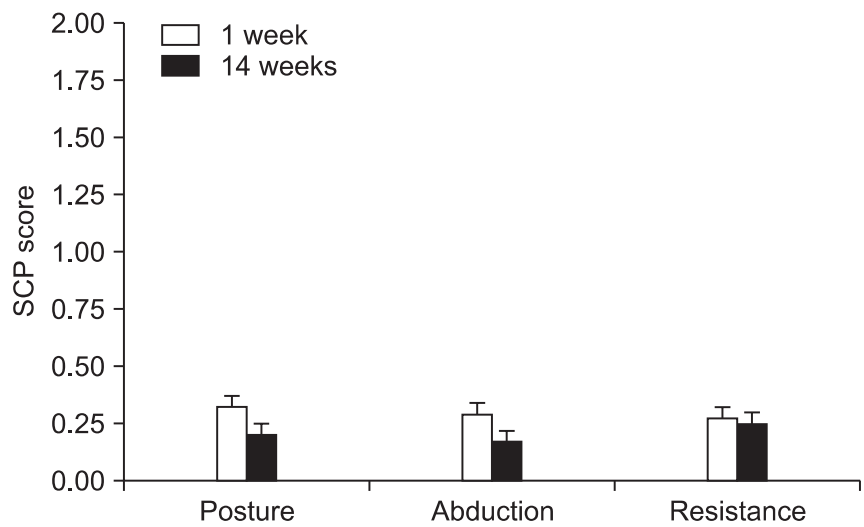

Fig. 2. Mean scores for the Scale for Contraversive Pushing (SCP) subscales (posture, abduction, resistance) at 1 and 14 weeks. (A) Experimental group and (B) control group. The values are expressed as mean \pm standard deviation.

Table 3. Somatosensory evoked potential (SEP) findings between two groups

\begin{tabular}{|c|c|c|c|c|c|c|c|}
\hline & & \multicolumn{3}{|c|}{1 week } & \multicolumn{3}{|c|}{14 weeks } \\
\hline & & Group I & Group II & p-value & Group I & Group II & p-value \\
\hline \multirow[t]{3}{*}{ Median SEPs } & Normal & 8 & 7 & 0.673 & 10 & 9 & 0.615 \\
\hline & Abnormal & 4 & 5 & & 2 & 3 & \\
\hline & No response & 0 & 0 & & 0 & 0 & \\
\hline \multirow[t]{3}{*}{ Tibial SEPs } & Normal & 7 & 8 & 0.673 & 9 & 10 & 0.615 \\
\hline & Abnormal & 5 & 4 & & 3 & 2 & \\
\hline & No response & 0 & 0 & & 0 & 0 & \\
\hline
\end{tabular}

Group I, patients with pusher syndrome; Group II, patients without pusher syndrome.

decreased from $5.7 \pm 1.6$ to $2.4 \pm 0.5$ in group I and from $4.6 \pm 1.1$ to $1.9 \pm 0.8$ in group II $(\mathrm{p}<0.05)$ (Table 2$)$.

\section{Comparison of SEP test}

The SEPs was conducted by stimulating the median nerve and the tibial nerve. No significant difference was found in the SEPs results of the two groups at 1 and 14 weeks after the stroke onset. In the 1st week test, abnormal results for the median nerve were obtained for 4 patients and for the tibial nerve for 5 patients in group I; similarly abnormal results were obtained for the median nerve in 5 patients and for the tibial nerve in 4 patients in group II (Table 3). In the 14th week test as well, abnormal results for the median nerve were observed for 2 patients and for the tibial nerve for 3 patients in group I. Further, abnormal results for the median nerve were observed for 3 patients and for the tibial nerve for 2 patients in group II (Table 3).

\section{DISCUSSION}

PS is a poorly understood complication in the hemiplegic patients, characterized by tilted posture, active pushing towards the affected body side, and resistance to passive correction of the incorrect posture [4]. Clinically, they are more likely to fall because they push their weight from the nonparetic side to the paretic side, and this misperception of the body orientation makes it difficult for the patients to move their weight and maintain the posture. This in turn causes problems in the rehabilitation process and lengthens the time taken to recover function [6]. Graviception is the perception of the body position, equilibrium, and direction of the gravitational forces [15]. The hypotheses that explain PS syndromes are based on visual-vestibular system impairment and misperception of body orientation in relation to gravity [15]. The subjective visual vertical is determined by asking the subjects, in complete darkness, to adjust a 
visible luminous line to what they consider to be the upright, earth vertical [12]. Recently, PS symptoms were found to be unrelated to semicircular canal abnormality; therefore, the visual-vestibular system impairment based hypothesis is not appropriate in explaining the PS symptoms [16]. As such, the distortion of the concept of subjective postural vertical (SPV) seems to be the main cause of PS. The SPV is assessed by asking the subjects, sitting on a tilting chair rotated in the frontal plane by the experimenter, to state in absence of vision when they feel their body as vertically orientated [12]. Karnath et al. [15] examined that when PS patients were seated on a chair tilted to one side with the eyes blinded, they perceived a position tilted by about $20^{\circ}$ toward the lesion as upright. This finding indicates that PS is associated with a severe misperception of the body orientation in relation to gravity, where efforts are made to compensate for this disparity in perception.

Davies [4] first described the association between the senses and PS and made an empirical remark that severe sensory impairment may trigger PS. Karnath [6] noted that the somatic senses may comprise sensory inputs in the gravity receptor system.

The SEPs can evaluate nerve cell injuries and recovery using a sensory nerve pathway [17]. It shows a sensory nerve pathway that reflects conduction though the dorsal columns of the spinal cord, synapsing in the dorsal column nuclei, and crossing in the opposite direction thought the medial lemniscus and thalamus to the parietal sensory cortex, thereby enabling objective evaluation of position and proprioception [18]. Attempts have been made to clarify the correlation between SEP test results and prognoses or brain lesions in stroke patients [19-21]. Kim et al. [22] reported that SEP test results showed good correlation with physical examination findings, and that SEP was associated with motor and sensory function. Further, Rhee et al. [18] noted that SEP was correlated with motor function. However, study on the association between SEP test results and PS is limited.

This study conducted the SEP test on stroke patients with and without PS, in order to examine the association between PS and somatosensory impairment. No significant differences were found between the two groups in SEP test results or CSII. This finding indicates that somatic senses may be an element of the sensory inputs, but are not significantly related to PS in terms of its cause. This result is consistent with that of Clement et al. [23] who found that the somatic senses are not particularly meaningful in PS, judging from the fact that when professional drivers and astronauts are instructed to keep their body perpendicular to the floor of the space station, the body leans forward by nearly $20^{\circ}$. Also, a patient with a complete sensory loss did not have any difficulties adjusting the SPV vertical in a lateral tilt chair [15]. It appears that somatosensory input plays a relatively minor role in the perception of the body posture, and that somatosensory loss does not necessarily result in PS. Although somatosense is an element of the sensory, it is not related to PS. For this reason, sensory impairment in PS patients may be a problem in the process of integrating the diverse senses, such as visual, superficial, and vestibular senses, to maintain a balance, or a problem with the accurate perception and cognition of the sensory information necessary for the planning and execution of the balance strategies. Meanwhile, a recent study has indicated that PS in the right-hemispheric lesion patients depends on the vestibular otolith input, suggesting a link between the system for postural control and the system responsible for processing vestibular otolith information [24].

At present, the commonly used treatment methods for PS [5] involve making the patients be aware of the misperception, repetitive performance of ADL upright, and training the patients to move their weight to the nonparetic side. However, a therapeutic target to ensure efficacy is lacking. Weakened muscle strength of the lower extremities, apraxia, and sensory impairment have been cited as possible causes of PS, but its precise cause has not been verified as yet $[9,15]$. Accordingly, further study on the sensory elements and causes that trigger altered proprioception in relation to gravity orientation is considered necessary for improving the efficacy of the PS treatment.

This study has several limitations. The number of subjects is not sufficient, as the incidence of PS is approximately $10 \%$ of all stroke patients. Furthermore, this study does not include an evaluation of other senses like the visual, superficial, and vestibular senses. Therefore, a study on the cause of PS involving a greater number of subjects that also compensates for other study limitations will contribute to the understanding of the mechanisms of PS and enable the development of more effective treatment methods. 
The pusher syndrome, a condition in which the stroke patients move their weight toward the paretic side when in the upright position, is known to occur because of the misperception of the body orientation in relation to gravity [4], and has a low clinical frequency. This study examined the association between PS and somatosensory impairment. The result showed that somatosenses did not have a significant correlation with PS as its cause. Additional study on the sensory input elements of the gravity receptor system as a cause of PS may help to develop effective treatment strategies for the stroke patients with PS.

\section{CONFLICT OF INTEREST}

No potential conflict of interest relevant to this article was reported.

\section{ACKNOWLEDGMENTS}

This study was supported by the research funds of Dong-A University.

\section{REFERENCES}

1. Stroke Unit Trialists' Collaboration. Organised inpatient (stroke unit) care for stroke. Cochrane Database Syst Rev 2007;(4):CD000197.

2. Dettmann MA, Linder MT, Sepic SB. Relationships among walking performance, postural stability, and functional assessments of the hemiplegic patient. Am J Phys Med 1987;66:77-90.

3. Spinazzola L, Cubelli R, Della Sala S. Impairments of trunk movements following left or right hemisphere lesions: dissociation between apraxic errors and postural instability. Brain 2003;126(Pt 12):2656-66.

4. Davies PM. Steps to follow: a guide to the treatment of adult hemiplegia. Berlin: Springer; 1985.

5. Karnath HO, Broetz D. Understanding and treating "pusher syndrome". Phys Ther 2003;83:1119-25.

6. Karnath HO. Pusher syndrome: a frequent but littleknown disturbance of body orientation perception. J Neurol 2007;254:415-24.

7. Danells CJ, Black SE, Gladstone DJ, McIlroy WE. Poststroke "pushing": natural history and relationship to motor and functional recovery. Stroke 2004;35:2873-8.
8. Karnath HO, Johannsen L, Broetz D, Ferber S, Dichgans J. Prognosis of contraversive pushing. J Neurol 2002;249:1250-3.

9. Karnath HO, Ferber S, Dichgans J. The neural representation of postural control in humans. Proc Natl Acad Sci U S A 2000;97:13931-6.

10. Bisdorff AR, Wolsley CJ, Anastasopoulos D, Bronstein AM, Gresty MA. The perception of body verticality (subjective postural vertical) in peripheral and central vestibular disorders. Brain 1996;119(Pt 5):1523-34.

11. Mittelstaedt H. Origin and processing of postural information. Neurosci Biobehav Rev 1998;22:473-8.

12. Paci M, Baccini M, Rinaldi LA. Pusher behaviour: a critical review of controversial issues. Disabil Rehabil 2009;31:249-58.

13. Deshpande N, Metter EJ, Ferrucci L. Validity of clinically derived cumulative somatosensory impairment index. Arch Phys Med Rehabil 2010;91:226-32.

14. Karnath HO, Brotz D, Gotz A. Clinical symptoms, origin, and therapy of the "pusher syndrome". Nervenarzt 2001;72:86-92.

15. Karnath HO, Ferber S, Dichgans J. The origin of contraversive pushing: evidence for a second graviceptive system in humans. Neurology 2000;55:1298-304.

16. Pontelli TE, Pontes-Neto OM, Colafemina JF, Araujo DB, Santos AC, Leite JP. Posture control in pusher syndrome: influence of lateral semicircular canals. Braz J Otorhinolaryngol 2005;71:448-52.

17. Kwon HK, Yim SK, Kim L, Chae SH, Lee HJ. Clinical usefulness of somatosensory evoked potentials in patients with stroke. J Korean Acad Rehabil Med 2003;27:355-60.

18. Rhee IG, Kim HS, Nah YS, Ahn KH, Lee YG. The relationship between SEP patterns and motor recovery in stroke patients. J Korean Acad Rehab Med 1987;11:194-9.

19. Feys H, Van Hees J, Bruyninckx F, Mercelis R, De Weerdt W. Value of somatosensory and motor evoked potentials in predicting arm recovery after a stroke. J Neurol Neurosurg Psychiatry 2000;68:323-31.

20. Gott PS, Karnaze DS, Fisher M. Assessment of median nerve somatosensory evoked potentials in cerebral ischemia. Stroke 1990;21:1167-71.

21. Vang C, Dunbabin D, Kilpatrick D. Correlation between functional and electrophysiological recovery in acute ischemic stroke. Stroke 1999;30:2126-30. 
22. Kim JH, Han TR, Lee CK. Median nerve somatosensory evoked potentials in hemiplegic patients. J Korean Acad Rehab Med 1987;11:119-29.

23. Clement G, Gurfinkel VS, Lestienne F, Lipshits MI, Popov KE. Adaptation of postural control to weight- lessness. Exp Brain Res 1984;57:61-72.

24. Baier B, Janzen J, Muller-Forell W, Fechir M, Muller N, Dieterich M. Pusher syndrome: its cortical correlate. J Neurol 2012;259:277-83. 